\title{
Estimating the filtration efficacy of cloth masks
}

\author{
Xinyu Mao $\odot^{1}$ and A. E. Hosoi $\circledast^{1,2}$ \\ ${ }^{1}$ Department of Mechanical Engineering, Massachusetts Institute of Technology, \\ Cambridge, Massachusetts 02139, USA \\ ${ }^{2}$ Department of Mathematics, Massachusetts Institute of Technology, \\ Cambridge, Massachusetts 02139, USA
}

(Received 15 March 2021; accepted 12 October 2021; published 5 November 2021)

\begin{abstract}
The current pandemic has spurred a great deal of debate about the use of cloth masks as effective alternatives to medical masks for the general public. Despite an abundance of experimental studies on the filtration of aerosols by cloth masks, there is currently a lack of analytical understanding to predict mask performance a priori. In this study, we establish a quantitative model for the pressure drop across woven heterogeneous cloth masks and their filtration efficiencies for aerosols with aerodynamic diameters less than $1 \mu \mathrm{m}$. To compare the intrinsic filtration capabilities of diverse materials, we introduce a filtration quality factor. Finally, we present a decision map to illustrate the tradeoffs between filtration efficiency and breathability and to provide practical guidance on the purchase of cloth masks. For the most commonly used cotton, polyester, and polypropylene masks, the mechanical filtration capability is fairly independent of the material, but it exhibits a high sensitivity to the porosity and fiber diameter. Additionally, keys to a high-quality cloth mask include a homogeneous structure, micro- or even nanofibrous materials, and a suitable mass per unit area of the textile.
\end{abstract}

DOI: 10.1103/PhysRevFluids.6.114201

\section{INTRODUCTION}

The COVID-19 pandemic has necessitated extensive use of face masks. Face masks have been shown to filter virus-laden droplets and aerosols generated by contagious people, and to protect healthy individuals from inhaling airborne aerosols, thus blocking transmission pathways of respiratory diseases [1,2]. Due to the shortage of medical masks and respirators early in the pandemic, health experts recommended cloth masks as an alternative [3]. Unlike medical masks and respirators, which are treated by electret charging techniques that activate masks to actively capture aerosols by electrostatic forces, common cloth masks passively filter aerosols mainly by diffusion, interception, inertial impaction, and gravitational settling [4]. In light of these different filtration mechanisms, it has been a topic of rigorous debate to what extent cloth masks can serve as effective filters for virus-laden aerosols [5], and which types of cloth masks are most effective to protect wearers.

Experimental studies investigating the filtration performance of common cloth masks have provided inconsistent results [6-11]. Cloth masks have been reported both to be comparable to N95 respirators [10,11] and to perform much worse [6-9]. Even for the same mask material, e.g., cotton, the measured data vary significantly among studies [6-11] (Fig. 1). These discrepancies could be due to any number of factors, including the large variety of different materials, fiber diameters, porosities, or fabric structures used in cloth masks.

Breathability and filtration efficiency are central to the quality of face masks. In this study, we refer to existing theories on the air permeability and filtration properties of fibrous networks. 
(a)

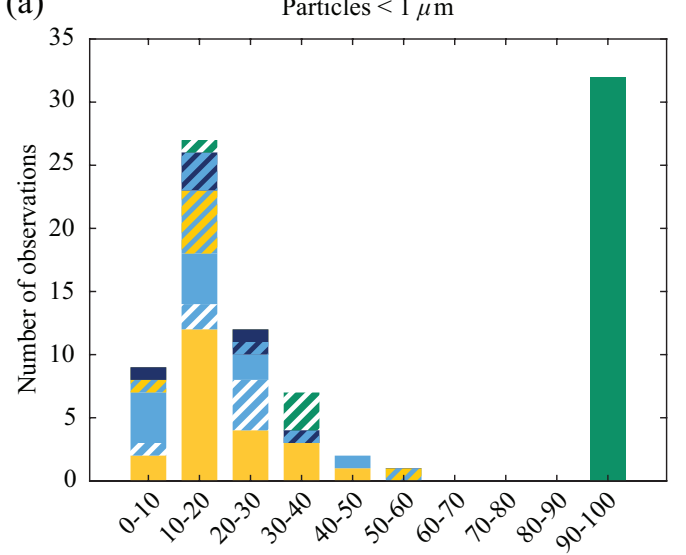

Filtration efficiency, $E(\%)$

cotton
poly-cotton blend (b) $\quad$ Particles $>1 \mu \mathrm{m}$

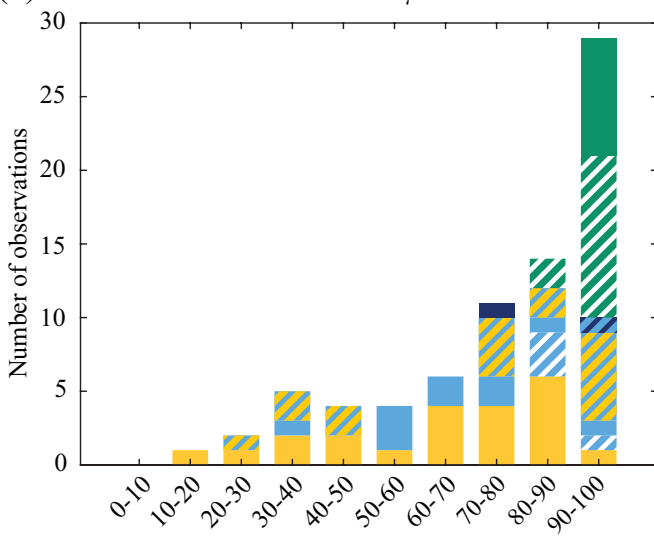

Filtration efficiency, $E(\%)$

polyester

polypropylene
2 surgical mask

N95 respirator

FIG. 1. Filtration efficiencies of common cloth masks and medical masks collected from the literature [7-9,12-19] and rated by the number of observations in different efficiency intervals. Filtered aerosol diameters are less than $1 \mu \mathrm{m}$ in (a) and greater than $1 \mu \mathrm{m}$ in (b). Each observation represents a specific type of mask from one publication. Note that this is intended as a qualitative snapshot to illustrate the wide range of observed efficiencies in the literature; test parameters including flow rate and particle size are not standardized across different publications.

For air permeability, previous studies provide analytical and empirical expressions of the pressure drop across fibrous networks, in which fibers can be randomly oriented in one [20-22], two [21-25], or three dimensions [21-23,25-27]. Air permeability of textiles has also been studied with classical fluid models [28-31] or neural networks [30]. However, structural diversity has so far prevented the formulation of a widely accepted model for the pressure drop across woven textiles. Existing filtration models are able to estimate the filtration efficiency of homogeneous filters or filters with a limited level of heterogeneity [32,33] which are applicable to nonwoven cloth masks. However, woven textiles are highly structured, heterogeneous fibrous networks and may contain gaps between threads, which significantly influences the airflow through the masks. Due to the strong coupling between the permeability and the filtration properties, aerosol filtration by woven cloth masks cannot be directly analyzed with existing filtration models. Furthermore, these models are not quite sufficient to guide material selection.

In this research article, we report a quantitative framework for estimating the pressure drop and filtration efficiency of cloth masks. Our primary interest is in the ability of cloth masks to protect healthy individuals from airborne aerosols. Specifically, we consider the measure of protection masks afford against aerosols with aerodynamic diameters less than $1 \mu \mathrm{m}$, a size range of significant importance for disease transmission [34]; in addition, we quantify the impact of the heterogeneous nature of woven textiles, which reflects the practical reality of most cloth masks. We first propose a general model for a first-order estimate of the pressure drop across cloth masks. The pressure drop model is then coupled with an adapted filtration model to compare the intrinsic filtration capabilities of several common materials for cloth masks. Finally, we establish a decision map to quantify the tradeoffs in selecting cloth masks during a pandemic. 


\section{PRESSURE DROP ACROSS CLOTH MASKS}

\section{A. Pressure drop across nonwoven textiles}

We begin by reviewing the classical model for homogeneous filters, in which fibers are randomly distributed in plane in order to approximate a nonwoven textile (e.g., a surgical mask). The pressure drop across a fibrous filter is generally formulated by Darcy's law [35]:

$$
\Delta P=\frac{\mu U Z}{\kappa} \quad \text { with } \quad \kappa=\frac{d_{f}^{2}}{4} \frac{1}{f(\alpha)},
$$

where $\mu$ is the air viscosity, $U$ is the face velocity, $Z$ is the thickness of the filter, and $\kappa$ is the permeability. Face velocity is defined as the average upstream fluid velocity relative to the filter. For homogeneous fibrous filters, $\kappa$ is a function of the fiber diameter $d_{f}$, the fiber volumetric ratio $\alpha$ (i.e., fiber packing density), and the spatial arrangement of fibers characterized by specific functional forms of $f(\alpha)$ [36]. For a random arrangement in the laminar, viscous flow regime, a popular empirical expression of $f(\alpha)$ was introduced by Davies in 1953 [24,32]:

$$
f_{\text {Dav }}(\alpha)=16 \alpha^{3 / 2}\left(1+56 \alpha^{3}\right) \text { for } 0.006<\alpha<0.3 \text {. }
$$

Fifty years later, Tomadakis and Robertson derived another expression of $f(\alpha)$, which shows a better agreement with experiments than that of Davies for $\alpha>0.3$ [22]:

$$
f_{\text {Tom }}(\alpha)=\left\{\frac{1-\alpha}{8 \ln ^{2}(1-\alpha)} \frac{\left(1-\alpha-\varepsilon_{p}\right)^{n+2}}{\left(1-\varepsilon_{p}\right)^{n}\left[(n+1)(1-\alpha)-\varepsilon_{p}\right]^{2}}\right\}^{-1},
$$

where $1-\alpha$ is the porosity of the filter, and constants $\varepsilon_{p}=0.11$ and $n=0.785$ for randomly overlapping fibrous structures. These two empirical relations, $f_{\text {Dav }}(\alpha)$ and $f_{\text {Tom }}(\alpha)$, are shown in Appendix A for comparison (Fig. 10).

Additionally, Eqs. (2) and (3) assume that the airflow around the fibers is a continuum, i.e., the Knudsen number $\mathrm{Kn}_{f}=2 \lambda / d_{f} \ll 1$, where $\lambda$ is the mean free path of the air molecules. This assumption holds true for many natural fibers and conventional synthetic fibers with $d_{f}$ on the order of $10 \mu \mathrm{m}$. However, for microfibers and nanofibers, the flow transits into the slip-flow regime, where the gas slip over the fibers leads to a reduction in $\Delta P$ [23,37]. This can be addressed by adding a correction factor $C_{\text {slip }}$ to Eq. (1). Among the studies on the pressure drop across nanofibrous filters, the relation introduced by Pich [38,39] and later Brown [40] is widely adopted [41-45], which gives $C_{\text {slip }}$ as [32]

$$
C_{\text {slip }}=\frac{1+1.996 \mathrm{Kn}_{f}}{1+1.996 \mathrm{Kn}_{f}\left[\frac{1}{1+\left\{2(1-\alpha)^{2} /\left(1-\alpha^{2}+2 \ln \alpha\right)\right\}}\right]} .
$$

See $C_{\text {slip }}$ as a function of $d_{f}$ for varying $\alpha$ in Appendix A (Fig. 11). Hence $\Delta P$ for homogeneous fibrous filters can be estimated as

$$
\Delta P=\frac{4 \mu U Z f(\alpha)}{d_{f}^{2}} C_{\text {slip }} \text { with } f(\alpha)= \begin{cases}f_{\mathrm{Dav}}(\alpha) & \text { for } 0.006<\alpha<0.3, \\ f_{\mathrm{Tom}}(\alpha) & \text { for } \alpha \geqslant 0.3 .\end{cases}
$$

\section{B. Pressure drop across woven textiles}

To extend this theory to pressure drop across a woven textile, consider the repeating unit illustrated in Fig. 2(b). We divide the repeating unit into gap-dominated and thread-dominated regions and set a uniform thickness $Z$ for the textile. The fraction of the gap-dominated area is denoted as $\varphi_{g}$. Most fibers are distributed within threads; however, gaps also contain a fraction of fibers, which may arise due to specific production techniques (e.g., brushing for flannel) or irregular shapes of fibers (e.g., cotton). Thus, we regard the gap and the thread as two types of porous media 
(a)

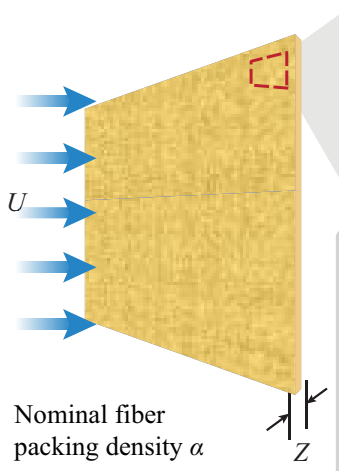

(b)

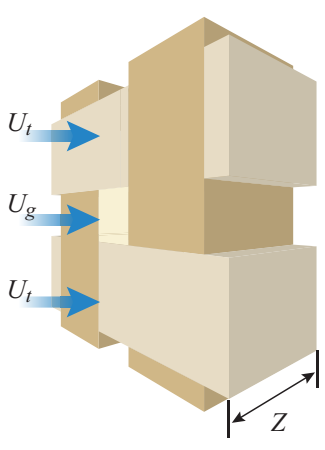

Gap-dominated area fraction $\varphi_{g}$ Gap fiber packing density $\alpha_{g}$

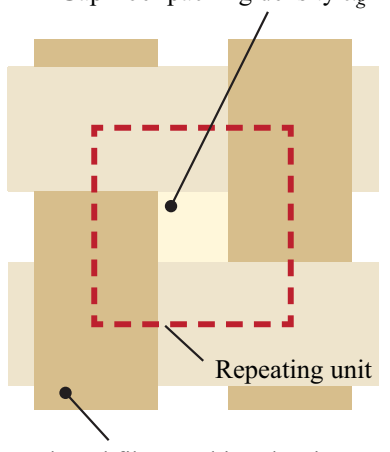

Thread fiber packing density $\alpha_{t}$

FIG. 2. (a) Schematics of a woven textile placed perpendicular to an airflow with face velocity $U$. (b) Magnified view of a repeating unit in (a) simplified as rectangular threads surrounding a square-shaped gap. The face velocities upstream of the threads and the gap are $U_{t}$ and $U_{g}$, respectively.

with different fiber packing densities. The nominal fiber packing density $\alpha$ is related to the thread fiber packing density $\alpha_{t}$ and gap fiber packing density $\alpha_{g}$ by

$$
\alpha=\alpha_{g} \varphi_{g}+\alpha_{t}\left(1-\varphi_{g}\right) .
$$

Hence, we select gap-dominated area fraction $\left(\varphi_{g}\right)$, fiber diameter $\left(d_{f}\right)$, thread fiber packing density $\left(\alpha_{t}\right)$, gap fiber packing density $\left(\alpha_{g}\right)$, and fabric thickness $(Z)$ as the five free parameters that describe a woven cloth mask.

Defining the pressure drops across the fabric, the threads, and the gaps as $\Delta P_{\text {textile }}, \Delta P_{t}$, and $\Delta P_{g}$, respectively, and noting that the pressure drops across the threads and the gaps must be the same, implies $\Delta P_{\text {textile }}=\Delta P_{t}=\Delta P_{g}$. The pressure drops $\Delta P_{t}$ and $\Delta P_{g}$ are calculated with Darcy's law, i.e.,

$$
\Delta P_{g}=\frac{\mu U_{g} Z}{\kappa_{g}} \quad \text { and } \quad \Delta P_{t}=\frac{\mu U_{t} Z}{\kappa_{t}},
$$

where $\kappa_{t}$ and $\kappa_{g}$ are the permeabilities of the thread-dominated and gap-dominated regions, respectively, and $U_{t}$ and $U_{g}$ are the nominal face velocities upstream of the two regions [see Fig. 2(b)], respectively. Conservation of mass provides the following relationship between the flow rates:

$$
U=U_{t}\left(1-\varphi_{g}\right)+U_{g} \varphi_{g}
$$

where $U$ is the average face velocity far upstream of the textile. Combining $\Delta P_{\text {textile }}=\Delta P_{t}=\Delta P_{g}$ and Eq. (8), the equivalent permeability of the textile $\kappa_{\text {textile }}$ can be written as a weighted average of $\kappa_{t}$ and $\kappa_{g}$ :

$$
\kappa_{\text {textile }}=\kappa_{t}\left(1-\varphi_{g}\right)+\kappa_{g} \varphi_{g}
$$

where $\kappa_{t}=d_{f}^{2} /\left[4 f\left(\alpha_{t}\right) C_{\text {slip }}\left(d_{f}, \alpha_{t}\right)\right]$ and $\kappa_{g}=d_{f}^{2} /\left[4 f\left(\alpha_{g}\right) C_{\text {slip }}\left(d_{f}, \alpha_{g}\right)\right]$. The pressure drop across the textile $\Delta P_{\text {textile }}$ can then be estimated as

$$
\Delta P_{\text {textile }}=\frac{\mu U Z}{\kappa_{\text {textile }}} .
$$

With $\Delta P_{\text {textile }}$ obtained, we can further calculate $U_{t}$ and $U_{g}$ with Eq. (7).

Variations in $\Delta P_{\text {textile }}$ normalized by the pressure drop $\Delta P$ across a homogeneous filter constructed of the same type of fiber and nominal fiber packing density $\alpha$ are shown in Fig. 3 . We choose $\alpha=0.3,0.4$, and 0.5 as the characteristic values for Figs. 3(a)-3(c), respectively, to mirror 
(a)

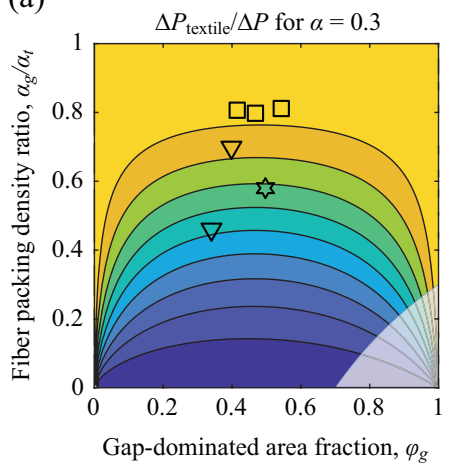

s twill weave trotin weave (b)

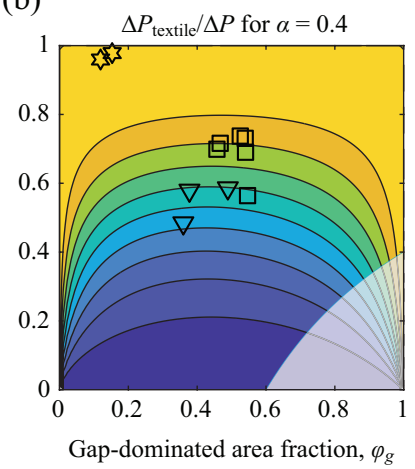

$\square$ poplin weave (c)

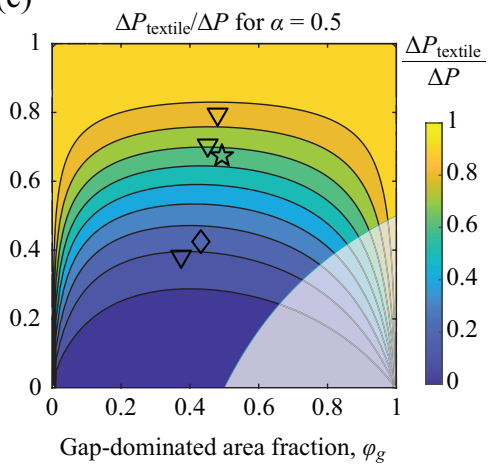

$\nabla$ plain weave

FIG. 3. Normalized pressure drop across textile $\Delta P_{\text {textile }} / \Delta P$ as a function of gap-dominated area fraction $\varphi_{g}$ and fiber packing density ratio $\alpha_{g} / \alpha_{t}$ for nominal fiber packing density (a) $\alpha=0.3$, (b) $\alpha=0.4$, and (c) $\alpha=$ 0.5 . Here, $\Delta P$ is the pressure drop across a homogeneous filter constructed of the same type of fiber and $\alpha$ as the woven textile. The gray shaded area corresponds to the inaccessible region described by Eq. (11). The experimental points showing typical values of $\varphi_{g}$ and fiber packing density ratio $\alpha_{g} / \alpha_{t}$ are obtained by analyzing data from Zangmeister et al. [9].

the typical range of $\alpha$ for woven cloth masks. When the fiber diameter $d_{f}$ and $\alpha$ are held constant, the normalized pressure drop $\Delta P_{\text {textile }} / \Delta P$ is a function of the fraction of the gap-dominated area $\varphi_{g}$ and the fiber packing density ratio $\alpha_{g} / \alpha_{t}$. For $\varphi_{g} \rightarrow 0, \varphi_{g} \rightarrow 1$, or $\alpha_{g} / \alpha_{t} \rightarrow 1$, since the textile approaches the homogeneous limit, $\Delta P_{\text {textile }} / \Delta P \rightarrow 1$. For an intermediate $\varphi_{g}$, because the permeability of gaps is typically higher than that of the threads, a significant amount of airflow bypasses the threads, leading to a lower $\Delta P_{\text {textile }} / \Delta P$. Additionally, $\Delta P_{\text {textile }} / \Delta P$ is mainly determined by the fiber packing density ratio $\alpha_{g} / \alpha_{t}$ at intermediate values of $\varphi_{g}$ (Fig. 3). Finally, note that the shaded area represents the condition

$$
\left[\frac{\alpha_{g}}{\alpha_{t}}\right] \varphi_{g}+\left(1-\varphi_{g}\right) \leqslant \alpha,
$$

which is inaccessible because the constraint $\alpha_{t}<1$ is violated.

To test the prediction in Eq. (9), we turn to the experimental data from Zangmeister et al. [9]. By extracting $\varphi_{g}, \alpha_{g}, \alpha_{t}$, and $d_{f}$ from textile images and assuming $Z$ on the order of the yarn width, we can analytically estimate the permeabilities of the woven textiles. The detailed method for the extraction of $\varphi_{g}, \alpha_{g}$, and $\alpha_{t}$ is described in Appendix B. Additionally, the fiber diameters of the tested textiles are on the order of $10 \mu \mathrm{m}$; thus, slip correction is negligible for estimating $\Delta P_{\text {textile }}$ (Fig. 11). Figure 4 shows the comparison between the analytical and experimental normalized permeabilities of textiles, $\kappa_{\text {textile }}^{(a)} / r_{f}^{2}$ and $\kappa_{\text {textile }}^{(e)} / r_{f}^{2}$, respectively, where $r_{f}=d_{f} / 2$ denotes the fiber radius. The error bars for the experimental permeabilities are due to the distributions of the estimated fiber radii from textile images. The analytical predictions generally agree with experimental measurements, and the small discrepancy between the analytical and experimental results can be partially attributed to the following factors. First, we assume a uniform thickness $Z$ for textiles, and we neglect local variations in $Z$ and the nominal $\alpha$. Second, we divide textiles into two types of porous media and treat each of them as homogeneous, which cannot fully capture the heterogeneity of textiles. Figure 4 provides a sense of the magnitude of the error in the estimation. Finally, to make a robust estimate of $\Delta P_{\text {textile }}$, Fig. 3 suggests that either $\varphi_{g}$ should be in the intermediate plateau region, or $\left[\varphi_{g}, \alpha_{g} / \alpha_{t}\right]$ should approach the homogeneous limit. We label in Fig. 3 the textiles we investigate by showing their extracted $\varphi_{g}$ and $\alpha_{g} / \alpha_{t}$ values. 


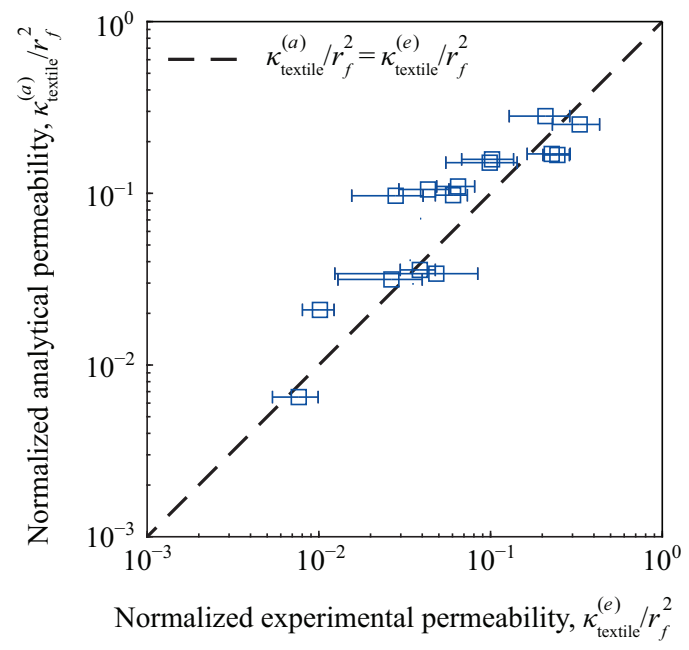

FIG. 4. Comparison between the analytical and experimental normalized permeabilities of textiles, $\kappa_{\text {textile }}^{(a)} / r_{f}^{2}$ and $\kappa_{\text {textile }}^{(e)} / r_{f}^{2}$, respectively, where $r_{f}$ denotes the fiber radius. The results are obtained by analyzing experimental data from Zangmeister et al. [9].

In addition to the double-porosity method described above, we also carried out a continuous permeability-map method to estimate $\Delta P_{\text {textile }}$ in Appendix $\mathrm{C}$, which provides close predictions to the results presented in Fig. 4.

\section{FILTRATION EFFICIENCY OF CLOTH MASKS}

\section{A. Filtration efficiency of nonwoven textiles}

As with the pressure drop analysis, we begin by reviewing filtration theory for nonwoven materials. The filtration efficiency is defined as the fraction of particles captured by the filter. In classical filtration theory, the filtration efficiency of a homogeneous fibrous filter $E$ is a function of fiber packing density $\alpha$, filter thickness $Z$, fiber diameter $d_{f}$, and single-fiber efficiency $\eta[33,36]$ :

$$
E=1-\exp \left(-4 \eta \frac{\alpha}{1-\alpha} \frac{Z}{\pi d_{f}}\right)
$$

where $\eta$ is the ratio between the number of particles collected by a single fiber and the number of incident particles in the projected area of the fiber [33].

The single-fiber efficiency is a combined effect of different particle collection mechanisms, including Brownian diffusion, interception, inertial impaction, gravitational settling, and possibly electrostatic attraction [33]. Different mechanisms dominate in different ranges of particle diameter $d_{p}$. Because we neglect the electrostatic attraction of cloth masks and focus on the range of $d_{p}<1$ $\mu \mathrm{m}$, diffusion and interception dominate, and other mechanisms are negligible [41]. Thus, $\eta$ is given by

$$
\eta=1-\left(1-\eta_{D}\right)\left(1-\eta_{R}\right)
$$

where $\eta_{D}$ represents single-fiber diffusion efficiency and $\eta_{R}$ is single-fiber interception efficiency. Most studies show that $\eta_{D}$ is proportional to $\mathrm{Pe}^{-2 / 3}$ [46], where Pe is the Péclet number. For example, Kirsch and Fuchs provided the empirical relation [47]

$$
\left.\eta_{D}\right|_{\text {Kir }}=2.7 \mathrm{Pe}^{-2 / 3}
$$


with $\mathrm{Pe}=d_{f} U / D$, where $D$ is the particle diffusivity. Additionally, $\eta_{R}$ is a function of the interception number $R=d_{p} / d_{f}$. A commonly used expression of $\eta_{R}$ was given by Liu and Rubow [48]:

$$
\left.\eta_{R}\right|_{\text {Liu }}=0.6 C_{r} \frac{1-\alpha}{\operatorname{Ku}(\alpha)} \frac{R^{2}}{1+R},
$$

where $C_{r}=1+1.996 \mathrm{Kn}_{f} / R$ is the correction factor due to the slip condition, and $\mathrm{Ku}(\alpha)=$ $-(\ln \alpha) / 2-3 / 4+\alpha-\alpha^{2} / 4$ is the Kuwabara number.

Unlike ideal homogeneous filters, $d_{f}$ for nonwoven textiles may be distributed in a certain range, and fibers can be clumped together. Thus, directly applying Eq. (12) tends to overestimate the filtration efficiency. To address these nonideal effects, Davies recommended replacing $d_{f}$ with an effective fiber diameter $d_{f(\text { eff })}$, which can be estimated by using the measured pressure drop across filters [32,33], i.e.,

$$
d_{f(\mathrm{eff})}^{2}=\frac{4 \mu U Z f(\alpha) C_{\text {slip }}\left(d_{f(\mathrm{eff})}, \alpha\right)}{\Delta P_{\text {textile }}} .
$$

\section{B. Filtration efficiency of woven textiles}

Following the double-porosity method described in Sec. II B and assuming aerosols are uniformly distributed in the airflow, the overall filtration efficiency of textile $E_{\text {textile }}$ can be written as a weighted average of the filtration efficiencies of the gap-dominated region, $E_{g}$, and the threaddominated region, $E_{t}$ :

$$
E_{\text {textile }}=E_{g} \frac{Q_{g}}{Q}+E_{t} \frac{Q_{t}}{Q},
$$

where $Q$ is the total flow rate through the textile, and $Q_{g}$ and $Q_{t}$ are the flow rates through the gaps and threads, respectively. With the velocities $U_{g}$ and $U_{t}$ calculated in Sec. II, $Q_{g} / Q=\varphi_{g} U_{g} / U$ and $Q_{t} / Q=\left(1-\varphi_{g}\right) U_{t} / U$.

To estimate $E_{g}$ and $E_{t}$, we adapt the classical filtration theory to each region of the textile. In analogy to Davies' method [Eq. (16)], given the measured pressure drop $\Delta P_{\text {textile, }}$, the effective fiber diameter $d_{f(\text { eff })}$ is implicitly determined via the following expression:

$$
d_{f(\text { eff })}^{2}=\frac{4 \mu U Z}{\Delta P_{\text {textile }}}\left\{\left[\frac{1-\varphi_{g}}{f\left(\alpha_{t}\right) C_{\text {slip }}\left(\alpha_{t}, d_{f(\text { eff })}\right)}\right]+\left[\frac{\varphi_{g}}{f\left(\alpha_{g}\right) C_{\text {slip }}\left(\alpha_{g}, d_{f(\text { eff })}\right)}\right]\right\}^{-1} .
$$

The actual fiber diameter $d_{f}$ is then replaced by $d_{f(\text { eff })}$ for the calculation of $\eta, E_{g}$, and $E_{t}$.

Next, the correlations given by Eqs. (14) and (15) are based on the assumption that aerosols will be captured as long as they contact the fibers [46]. Thus, the influence of the material type is not reflected in previous models. Moreover, the nonstandard cross-sectional shapes of many natural fibers may contribute to a deviation from the theoretical predictions. To include these factors, we generalize the Kirsch and Fuchs relation and assume

$$
\eta_{D}=\beta \mathrm{Pe}^{-2 / 3} .
$$

Here, $\beta$ is a diffusion filtration quality factor that characterizes the capability of a material to capture aerosols by diffusion. The interception efficiency $\eta_{R}$ can be modified in a similar manner. To isolate the influence of material type on the (diffusional) filtration efficiency, we employ the following method to extract $\beta$ from experimental data: when $d_{f}$ is held constant, decreasing the particle diameter $d_{p}$ leads to a simultaneous decrease in Pe and $R$, and eventually $\eta \sim \eta_{D}=\beta \mathrm{Pe}^{-2 / 3} \gg \eta_{R}$. Thus, in the limit of small particles, by replacing Eq. (14) with Eq. (19) for the calculation of $E_{g}$ and $E_{t}, \beta$ can be implicitly determined with

$$
1-E_{\text {textile }}=\exp \left(\beta \mathrm{Pe}_{g}^{-2 / 3} \mathcal{L}_{g}\right) \bar{Q}_{g}+\exp \left(\beta \mathrm{Pe}_{t}^{-2 / 3} \mathcal{L}_{t}\right) \bar{Q}_{t},
$$


(a)

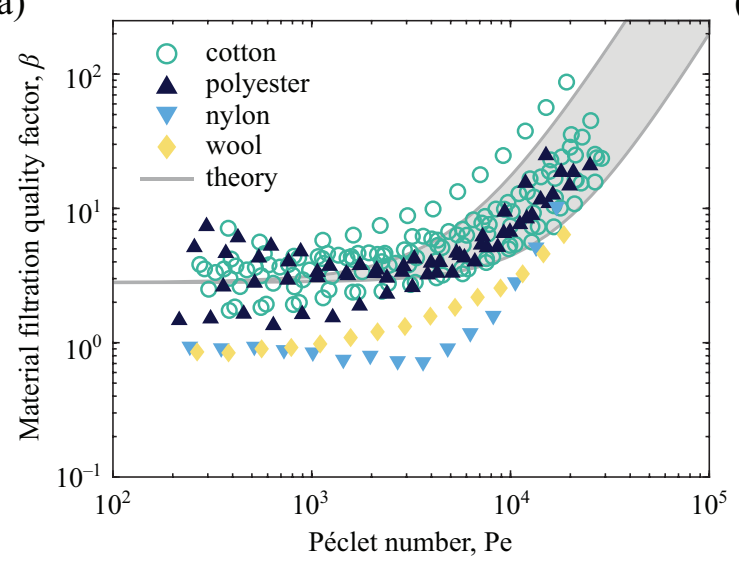

(b)

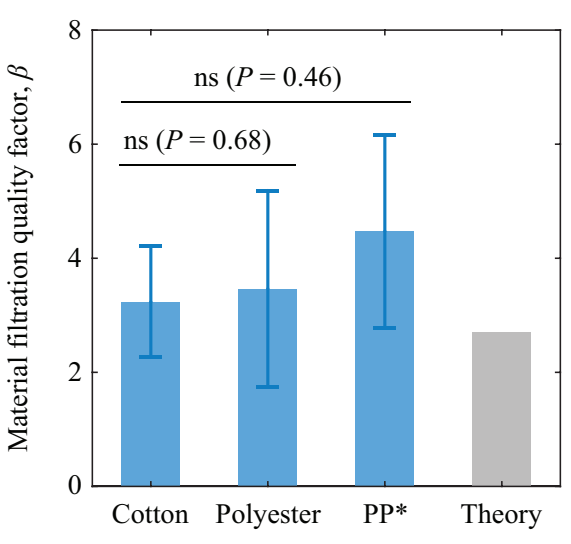

FIG. 5. (a) Diffusion filtration quality factor $\beta$ as a function of the Péclet number Pe for woven cloth masks. The theoretical curves correspond to modeling results for Eqs. (14) and (15) with $d_{f}$ ranging from 10 to 20 $\mu \mathrm{m}$. The experimental results are obtained by analyzing data from Zangmeister et al. [9]. (b) Comparison of $\beta$ values at the low-Pe limit between theory and the three most common mask materials. An asterisk denotes results for polypropylene (PP) that are derived from Jackiewicz and Werner [49]. The $P$-value is determined by the one-way ANOVA test; ns denotes not significant.

where $\mathcal{L}_{g}=-4\left[\alpha_{g} /\left(1-\alpha_{g}\right)\right]\left[Z /\left(\pi d_{f(\mathrm{eff})}\right)\right], \mathcal{L}_{t}=-4\left[\alpha_{t} /\left(1-\alpha_{t}\right)\right]\left[Z /\left(\pi d_{f(\mathrm{eff})}\right)\right], \bar{Q}_{g}=\varphi_{g} U_{g} / U$, and $\bar{Q}_{t}=\left(1-\varphi_{g}\right) U_{t} / U$. For a specific filter, as $d_{p}$ decreases, we expect $\beta$ to asymptote to a constant at the low-Pe limit. To test this hypothesis, we again analyze the experimental data of Zangmeister et al. [9] and show the $\beta$-Pe relation for four mask materials in Fig. 5(a). A theoretical curve derived from Eqs. (14) and (15) is also shown for comparison. Note that the diffusion-dominated regime corresponds to $\mathrm{Pe}<10^{3}$, and $\beta$ indeed approaches a constant value as $\mathrm{Pe}$ decreases for each tested mask. The scattered data for cotton and polyester is a result of the uncertainty in $\alpha$ due to the uncertainty in the estimated thickness $Z$ of each mask. Distributions of $\beta$ for cotton and polyester are both close to the theoretical curve, whereas nylon shows considerably lower $\beta$ values. This suggests that nylon fabrics may not be strong candidates for cloth masks, which has also been observed by Zhao et al. [8]. To further compare the filtration capabilities of the most commonly used mask materials, Fig. 5(b) shows extracted $\beta$ values at the low-Pe limit for cotton, polyester, and polypropylene based on experimental studies [9,49]. No significant difference in $\beta$ is found for these three materials, which are all close to the theoretical prediction. The results also suggest that polypropylene may have a slight advantage over cotton and polyester in terms of mechanical filtration performance.

We now compare $E_{\text {textile }}$ with the filtration efficiency $E$ of a homogeneous filter constructed of the same type of fiber and nominal $\alpha$. Figure 6(a) shows $E_{\text {textile }} / E$ as a function of $\varphi_{g}$ and $\alpha_{g} / \alpha_{t}$ at typical mask parameter values. Similar to Fig. 3, $E_{\text {textile }} / E \rightarrow 1$ when the textile approaches the homogeneous limit, i.e., $\varphi_{g} \rightarrow 0, \varphi_{g} \rightarrow 1$, or $\alpha_{g} / \alpha_{t} \rightarrow 1$. At an intermediate $\varphi_{g}, E_{\text {textile }} / E$ is relatively low because of the low filtration efficiencies in the gaps $E_{g}$ and the high relative flow

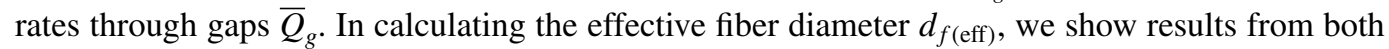
Eqs. (18) and (16) and present the corresponding $E_{\text {textile }} / E$ results with solid and dashed contour lines, respectively [Fig. 6(a)]. Despite some differences, especially at high $\varphi_{g}$ values, both the double-porosity method and Davies' method capture the influence of $\varphi_{g}$ and $\alpha_{g} / \alpha_{t}$ on the filtration efficiency of woven textiles. Additionally, the textile examples in Fig. 6(a) indicate that $E_{\text {textile }} / E$ is observed to be as low as 0.4 for plain weaves due to the effect of gaps. Denser weaves such as poplin tend to perform better with $E_{\text {textile }} / E$ approximately 0.7 . 
(a)

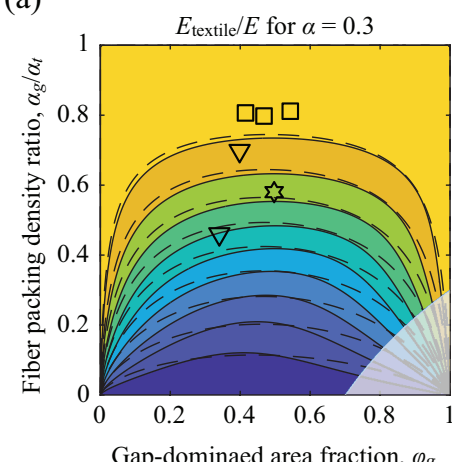

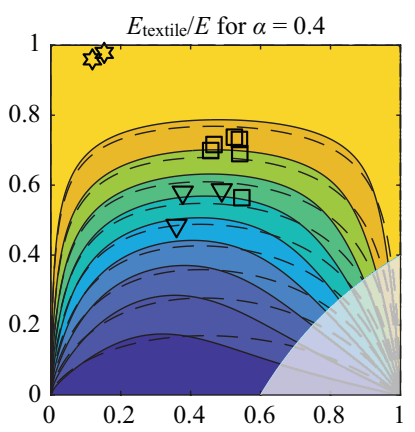

Gap-dominaed area fraction, $\varphi_{g}$

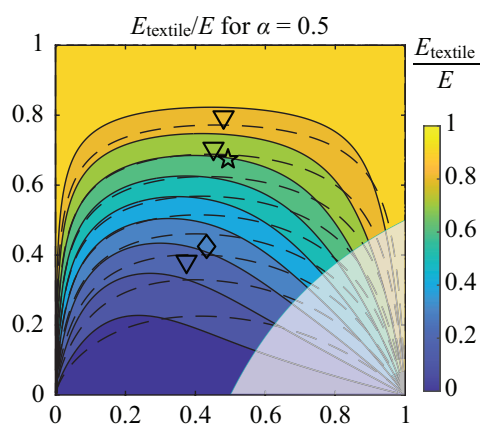

Gap-dominaed area fraction, $\varphi_{g}$
\& twill weave is satin weave
$\diamond$ sateen weave
$\square$ poplin weave
$\nabla$ plain weave _ - _ Davies' method
$\square$ inaccessable

(b)

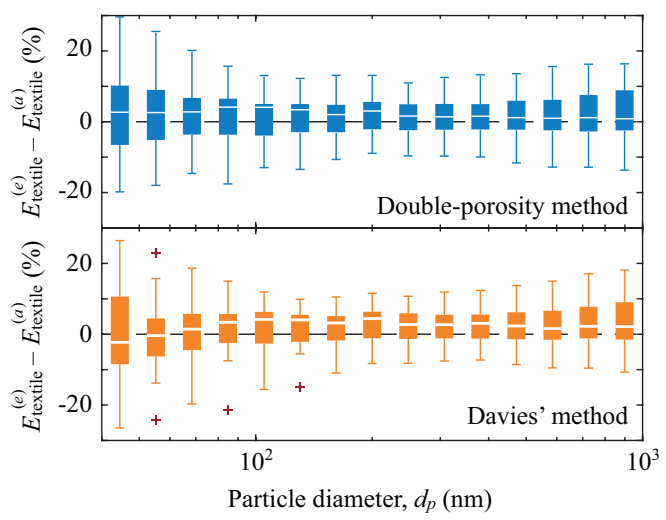

(c)

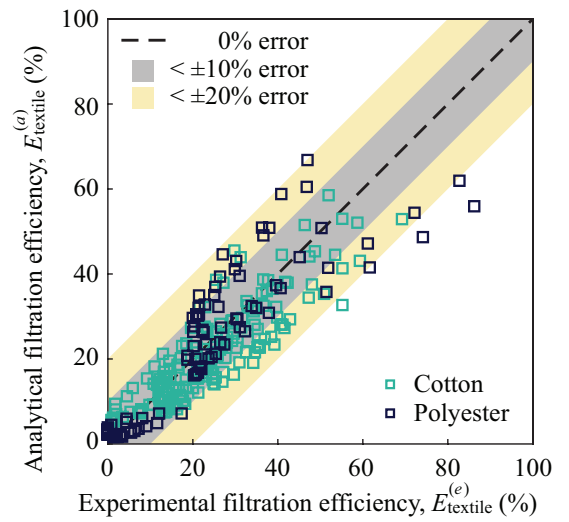

FIG. 6. (a) Normalized filtration efficiency of woven textile $E_{\text {textile }} / E$ as a function of gap-dominated area fraction $\varphi_{g}$ and fiber packing density ratio $\alpha_{g} / \alpha_{t}$ for nominal fiber packing density $\alpha=0.3,0.4$, and 0.5 . $E$ is the filtration efficiency of a homogeneous filter constructed of the same type of fiber and $\alpha$ as the woven textile. The solid and dashed contour lines are based on the double-porosity method [Eq. (18)] and the Davies' method [Eq. (16)], respectively. Here, $d_{f}=10 \mu \mathrm{m}, d_{p}=300 \mathrm{~nm}, U=5.3 \mathrm{~cm} / \mathrm{s}$, and $Z=0.5 \mathrm{~mm}$. (b) Box-andwhisker plot for the difference between the experimental and analytical mask filtration efficiencies $\left(E_{\text {textile }}^{(e)}-\right.$ $E_{\text {textile }}^{(a)}$ ) for varying particle diameters $d_{p}$. (c) $E_{\text {textile }}^{(e)}$ vs $E_{\text {textile }}^{(a)}$ for cotton and polyester masks based on the double-porosity method.

To test the prediction in Eq. (17), we analytically calculate the filtration efficiencies of various cotton and polyester masks and compare the results with the experimental data from Zangmeister et al. [Figs. 6(b) and 6(c)]. Note that for conventional fibers with $d_{f}$ on the order of $10 \mu \mathrm{m}$, slip correction can be neglected in calculating $d_{f \text { (eff) }}$ [Eqs. (18) and (16)]. In calculating $\eta_{D}$, the $\beta$-values are analyzed from a different source (Zhao et al. [8]), where $\beta_{\text {cotton }}=2.97$ and $\beta_{\text {polyester }}=2.64$. Figure 6(c) shows that $80 \%$ of the efficiency predictions have an error less than $10 \%$, and $98 \%$ of the predictions have an error less than $20 \%$.

Finally, our analytical model is based on ideal experimental setups in which both textile and aerosols are charge-neutral. Thus, the effect of electrostatic interactions between fibers and aerosols is negligible. However, electrostatic interactions can be significant for newly generated charged aerosols [33]. Although aerosols will eventually be neutralized as a whole in the steady-state, a fraction of aerosols still carry opposite charges due to random collisions with ions in the air [33]. For these cases, additional terms may be necessary to characterize electrostatic interactions in 
TABLE I. Variations in the pressure drop $\Delta P_{\text {textile }}$ across a woven cloth mask and its filtration efficiency $E_{\text {textile }}$ due to the variation in a single mask parameter, while other mask parameters are held constant. The upward and downward arrows denote an increase and a decrease in parameter values, respectively.

\begin{tabular}{lcc}
\hline \hline & \multicolumn{2}{c}{ Mask performance } \\
\cline { 2 - 3 } Mask parameters & Pressure drop $\Delta P_{\text {textile }}$ & Filtration efficiency $E_{\text {textile }}$ \\
\hline Gap-dominated area fraction $\varphi_{g}, \uparrow$ & $\downarrow$ & $\downarrow$ \\
Fiber diameter $d_{f}, \uparrow$ & $\downarrow$ & $\downarrow$ \\
Thread fiber packing density $\alpha_{t}, \uparrow$ & $\uparrow$ & $\uparrow$ \\
Gap fiber packing density $\alpha_{g}, \uparrow$ & $\uparrow$ & $\uparrow$ \\
Mask thickness $Z, \uparrow$ & $\uparrow$ & $\uparrow$ \\
\hline \hline
\end{tabular}

Eq. (13), which give rise to higher single fiber efficiencies. This suggests that our model provides a conservative estimate for the filtration efficiency of cloth masks in the natural state.

\section{PRACTICAL GUIDANCE ON SELECTING CLOTH MASKS}

Given the estimates in Secs. II and III, we now have sufficient information to provide some practical guidance on the selection of cloth masks. First, by varying each free parameter involved in our analysis, i.e., $\varphi_{g}, d_{f}, \alpha_{t}, \alpha_{g}$, or $Z$ for woven masks, we observe simultaneous increases or decreases in the pressure drop and filtration efficiency (Table I); i.e., there is no free lunch: an increase in breathability always corresponds to a decrease in filtration efficiency, and vice versa. Thus, consumers have to make a tradeoff between the breathability and filtration efficiency in selecting masks.

To quantify this tradeoff, the quality factor (QF) has been widely adopted for comparison of different filters [33]:

$$
\mathrm{QF}=\frac{-\ln \left(1-E_{\text {textile }}\right)}{\Delta P_{\text {textile }}},
$$

where QF represents the ratio between the filtration efficiency and the pressure drop per unit thickness for a differentially thin layer [33]. A higher QF corresponds to better filtration performance. To understand the underlying factors that determine QF and apply the analysis to cloth masks, we insert the expressions for $E_{\text {textile }}$ and $\Delta P_{\text {textile }}$ in Eq. (21). To perform a unified analysis for both woven and nonwoven textiles, we first assume that gaps will not induce an anomalously low $\delta P:=\Delta P_{\text {textile }} / \Delta P$ in a realistic woven mask. For simplicity, we assume $\delta P>0.5$, which is valid for the majority of masks we investigate (Fig. 3). Second, we employ Davies' method to analyze filtration efficiency. A deterministic relationship for $\mathrm{QF}$ reads

$$
\mathrm{QF}=\frac{1}{\pi \mu U \delta P} \frac{\alpha}{1-\alpha} \frac{d_{f}^{2}}{d_{f(\mathrm{eff})}} \frac{\eta_{\min }\left(d_{f(\mathrm{eff})}, \alpha\right)}{f(\alpha) C_{\mathrm{slip}}\left(d_{f}, \alpha\right)},
$$

where $\eta_{\min }$ is obtained at the most penetrate particle size (MPPS); MPPS is on the order of $0.1-0.5$ $\mu \mathrm{m}$ at typical face velocities for face masks [50]. When $U$ is held constant, QF is determined by fiber diameter $d_{f}$ and fiber packing density $\alpha$ [Eq. (22)]. Figure 7 shows the variations in QF for $d_{f}$ varying from $10^{-3}$ to $20 \mu \mathrm{m}$ and $\alpha$ varying from $10^{-2}$ to 0.7 . For a specific $\alpha$, there is an optimal fiber diameter $d_{f}$ that corresponds to a maximal QF (dashed line in Fig. 7). However, the optimal $d_{f}$ is considerably smaller than what is practically realizable in most textiles. Additionally, the classical filtration theory may not suffice to accurately model aerosol filtration in the free molecular flow regime $\left(\mathrm{Kn}_{f}>10\right.$ [51]; Fig. 7). Given these caveats, we focus our investigation on the parameter regime $d_{f}>0.3 \mu \mathrm{m}$ [52] (unshaded region in Fig. 7). In this regime for most textiles, QF increases as $d_{f}$ or $\alpha$ decreases. Specifically, the increase in $\eta_{\min }$ counterbalances the decrease in $d_{f}$ in Eq. (22), 


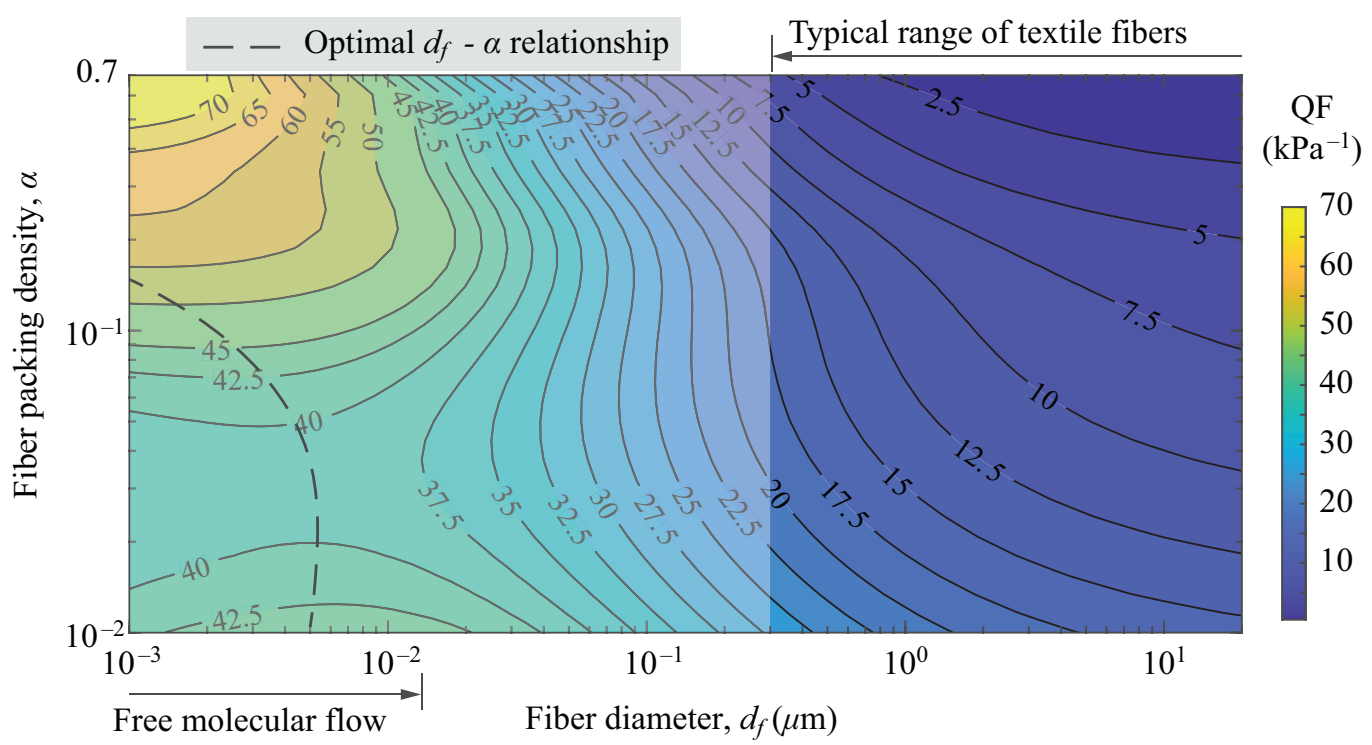

FIG. 7. Quality factor (QF) as a function of fiber diameter $d_{f}$ and fiber packing density $\alpha$. Here, $U=5.3$ $\mathrm{cm} / \mathrm{s}, \delta P=0.5$, and $\beta=3.1$.

thus leading to an increase in QF. Therefore, the optimal cloth masks should be made of ultrafine fibers at a low fiber packing density.

In the following, we provide practical guidance on the selection of cloth masks based on easily accessible parameters for consumers. We consider a scenario in which the objective is to determine a set of mask parameters that can guarantee a filtration efficiency of no less than $E_{\min }$ for a whole spectrum of particle size $d_{p}$, with the constraint that the pressure drop should be lower than a maximal tolerated value $\Delta P_{\max }$ at a typical face velocity $U$. Since most vendors do not provide a complete set of mask parameters such as $\alpha$ and $Z$, we perform some conversions and simplifications. First, we perform a unified analysis for both woven and nonwoven textiles. Again, we employ Davies' method to analyze filtration efficiency and assume $\delta P>0.5$. Second, we replace $Z$ with the grammage $G$, a metric that is more easily accessible and is defined as the mass per unit area of the textile in $\mathrm{g} / \mathrm{m}^{2}$. The grammage $G$ and the thickness $Z$ are related by

$$
G=\rho \alpha Z
$$

for $\rho$ in $\mathrm{g} / \mathrm{m}^{3}$. Thus, the problem can be formulated as follows: given $U, E_{\min }$, and $\Delta P_{\max }$, choose $d_{f}, G$, and $\alpha$, such that

$$
\Delta P_{\text {textile }} \leqslant \Delta P_{\max }, \quad E \geqslant E_{\min }, \quad \text { and } Z \leqslant Z_{\max }
$$

The third constraint in Eq. (24) limits the mask thickness to practical values. Based on test standards for $U$ and $\Delta P_{\max }$ [53] and common sense for $Z_{\max }$, we set $U=0.053 \mathrm{~m} / \mathrm{s}$ [7], $\Delta P_{\max }=50 \mathrm{~Pa}$, and $Z_{\max }=0.01 \mathrm{~m}$.

Next, Eq. (24) is recast with the accessible mask parameters. By inserting Eqs. (5) and (23) into Eq. (24), the pressure constraint is equivalent to

$$
G \leqslant A_{1} \frac{\alpha}{f(\alpha) C_{\text {slip }}\left(d_{f}, \alpha\right)} \quad \text { with } A_{1}=\frac{\rho \Delta P_{\max } d_{f}^{2}}{4 \delta P \mu U}
$$


(a)

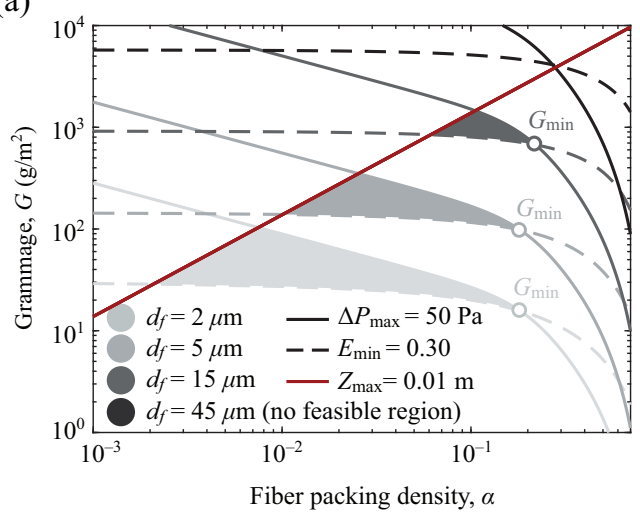

(b)

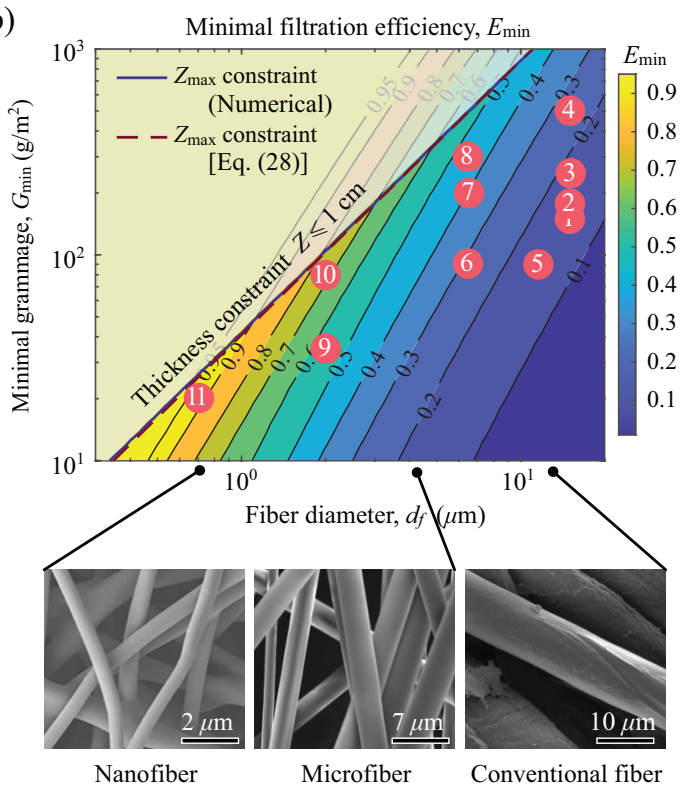

FIG. 8. (a) Feasible values of grammage $G$ and fiber packing density $\alpha$ of polyester cloth masks shown in the shaded gray regions, which correspond to varying fiber diameter $d_{f}$. The masks are subject to constraints $E_{\min }=0.30, \Delta P_{\max }=50 \mathrm{~Pa}$, and $Z_{\max }=0.01 \mathrm{~m} . G_{\min }$ is the minimal grammage that can satisfy these constraints. (b) Minimal filtration efficiency $E_{\min }$ as a function of fiber diameter $d_{f}$ and grammage $G_{\min }$ for a polyester cloth mask. The solid line corresponds to $Z \leqslant Z_{\max }$, and the dashed line represents the analytical estimate Eq. (28). The circled numbers demonstrate characteristic parameter values of cloth mask candidates. Microscopic images by Alarifi et al. [54] for nanofibers, Martín et al. [55] for microfibers, and Grgac et al. [56] for conventional fibers. Images of microfibers and conventional fibers are adapted under the terms of the CC-BY Creative Commons Attribution 4.0 International license. For both (a) and (b), $U=5.3 \mathrm{~cm} / \mathrm{s}$ and $\rho=1380 \mathrm{~kg} / \mathrm{m}^{3}$.

For the efficiency constraint, by inserting Eqs. (12) and (23) (substitute $d_{f}$ with $d_{f \text { (eff) }}$ ) into Eq. (24), we obtain

$$
G \geqslant A_{2} \frac{1-\alpha}{\eta_{\min }\left(d_{f(\mathrm{eff})}, \alpha\right)} \quad \text { with } A_{2}=\frac{\pi}{4} \rho d_{f(\mathrm{eff})} \ln \frac{1}{1-E_{\min }} .
$$

Finally, the thickness constraint is equivalent to

$$
G \leqslant \rho \alpha Z_{\max } .
$$

Four groups of representative solutions for Eqs. (25)-(27) are shown in Fig. 8(a). For a specific $d_{f}$, the gray area indicates the region where the three constraints are satisfied and constitute a feasible region for cloth masks. Notably, the intersection point between the pressure and efficiency constraints [Eqs. (25) and (26)] indicates both a minimal grammage $G_{\min }$ and a minimal thickness $Z_{\min }$ that meet the three constraints. Additionally, the feasible region shrinks to a point at a critical $d_{f}$, beyond which no solution can simultaneously satisfy the three constraints in Eq. (24) [Fig. 8(a)].

By integrating the results in Fig. 8(a) for different combinations of parameter values, a decision map based on $G_{\min }, d_{f}$, and $E_{\min }$ is illustrated in Fig. 8(b). From the contour map, one can identify the combinations of $G_{\min }$ and $d_{f}$ that fulfill a specific $E_{\min }$. It is evident that smaller fiber diameters $d_{f}$ allow for lower minimal grammages $G_{\min }$ to meet a given efficiency standard. Because $d_{f}$ of cotton fibers is generally around $10-20 \mu \mathrm{m}$, cotton textiles with a grammage lower than $1000 \mathrm{~g} / \mathrm{m}^{2}$, including bandanas, T-shirts, conventional bed sheets, and bath towels, can only reach minimal 
filtration efficiencies of 0.4 or less. With a low grammage and a similar fiber diameter to fine cotton fibers, silk napkins also provide limited protection against aerosols. Microfiber textiles with $d_{f}=6$ $\mu \mathrm{m}$ can achieve $E_{\min }=0.5$. Meltblown textiles have even finer fibers with $d_{f}$ around 2-4 $\mu \mathrm{m}$, leading to $E_{\min }$ as high as 0.7 . A nanofibrous electrospun face mask can achieve $E_{\min }>0.8$ with $G_{\min } \sim 20 \mathrm{~g} / \mathrm{m}^{2}$, exceeding the performance of other common textiles. Meltblown and electrospun textiles [e.g., Textiles 9-11 in Fig. 8(b)] have significant advantages over most woven textiles that have larger fiber diameters [e.g., Textiles $1-8$ in Fig. 8(b)].

Furthermore, based on the condition $Z_{\min } \leqslant Z_{\max }$, the thickness constraint $Z_{\max } \leqslant 0.01 \mathrm{~m}$ is illustrated in Fig. 8(b). The thickness constraint poses higher restrictions on $G_{\min }$ for finer fibers, because a lower $\alpha$ is required to avoid surpassing the pressure constraint. In the parameter space of Fig. 8(b), numerical results reveal that $\alpha$ is less than 0.1 near the thickness constraint line. Thus, by using $f_{\text {Dav }}(\alpha)$ and equating Eqs. (25) and (27), a first-order estimate for the thickness constraint in the form of a $G_{\min }-d_{f}$ relation reads

$$
G_{\min } \leqslant\left[\frac{1}{16} \rho\left(\frac{\Delta P_{\max }}{\delta P \mu U}\right)^{2 / 3} Z_{\max }^{1 / 3}\right] d_{f}^{4 / 3},
$$

which is illustrated in Fig. 8(b) as the dashed line.

\section{Examples of cloth mask design}

In addition to providing practical guidance for mask consumers, our model can also guide manufacturers through the design of mask materials. Here we discuss two example scenarios to illustrate the design process for two different types of manufacturing capabilities.

In the first scenario, manufacturers produce fabrics in-house. As discussed above, our foremost piece of advice is to make the fiber diameter $d_{f}$ as small as possible. Then, the manufacturer selects desired mask efficacies $\Delta P_{\max }$ and $E_{\min }$. Following the procedure introduced in Eqs. (24)-(27), the manufacturer constructs a feasible graph [Fig. 8(a)] which provides feasible combinations of grammage $G$ and fiber packing density $\alpha$ (gray shaded regions). For example, assume Manufacturer A plans to produce polyester masks with $d_{f}=5 \mu \mathrm{m}$ that can achieve $\Delta P_{\max }=50 \mathrm{~Pa}$ and $E_{\min }=$ 0.30. From Fig. 8(a), they readily find feasible parameters $G=200 \mathrm{~g} / \mathrm{m}^{2}$ and $\alpha=0.06$, which corresponds to a fabric thickness of $Z=2.4 \mathrm{~mm}$ [Eq. (23)].

In the second scenario, manufacturers procure fabrics from external suppliers and must select one type of fabric from multiple candidates. For this scenario, Secs. II and III provide strategies

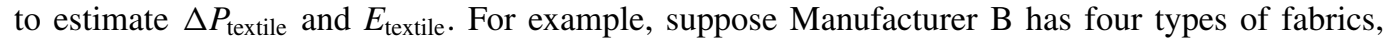
Fabrics A-D, and knows their compositions and grammages (Table II). After analyzing images of each fabric following Appendix B, Manufacturer B obtains parameters $Z, d_{f}, \varphi_{g}, \alpha_{t}$, and $\alpha_{g}$. The pressure drop $\Delta P_{\text {textile }}$ can then be calculated with Eqs. (9) and (10). By inserting $\Delta P_{\text {textile }}$ into

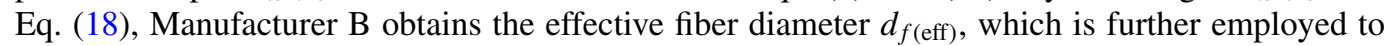
determine $E_{g}, E_{t}$, and $E_{\text {textile }}$ following Sec. III [Eqs. (12), (13), (15), (17), and (19)]. Finally, a scatter plot can be constructed showing the tradeoff between filtration efficiency and breathability for Fabrics A-D (Fig. 9). Now suppose Manufacturer B hopes to produce cloth masks with desired properties $\Delta P_{\max }=80 \mathrm{~Pa}$ and $E_{\min }=35 \%$. It is evident that Fabric $\mathrm{C}$ is the best candidate (Fig. 9).

\section{CONCLUSIONS}

In summary, we establish a quantitative model to predict the breathability and filtration efficiency of cloth masks and test the validity of our model with previous experimental results reported in the literature. We find that there is no significant difference in the diffusion filtration quality factor $\beta$ for cotton, polyester, and polypropylene. Thus, the material type may not be the most important factor in selecting masks. Rather, our practical decision map indicates that ultrafine fibers such as nanofibers and microfibers can effectively enhance the filtration efficiency of cloth masks at a low grammage. Due to larger fiber diameters and gaps in weave, woven textiles typically have low 
TABLE II. Estimation of filtration efficacies of four candidate fabrics for Manufacturer B. Asterisks denote mask properties analyzed from fabric images. Here, $U=5.3 \mathrm{~cm} / \mathrm{s}$, and $E_{\text {textile }}$ is obtained at MPPS.

\begin{tabular}{lcccc}
\hline \hline & & & Fabric $\mathrm{C}$ & Fabric $\mathrm{D}$ \\
& $\begin{array}{c}\text { Fabric A } \\
100 \% \text { cotton }\end{array}$ & $\begin{array}{c}\text { Fabric B } \\
100 \% \text { polyester }\end{array}$ & $\begin{array}{c}100 \% \\
\text { polypropylene }\end{array}$ & $\begin{array}{c}35 \% \text { cotton/ } \\
65 \% \text { polyester }\end{array}$ \\
\hline Grammage, $G\left(\mathrm{~g} / \mathrm{m}^{2}\right)$ & 150 & 140 & 100 & 200 \\
Material density, $\rho\left(\mathrm{kg} / \mathrm{m}^{3}\right)$ & 1550 & 1380 & 920 & 1440 \\
* Fabric thickness, $Z(\mathrm{~mm})$ & 0.30 & 0.25 & 0.50 & 0.50 \\
Nominal fiber packing density, $\alpha$ & 0.32 & 0.41 & 0.22 & 0.28 \\
* Fiber diameter, $d_{f}(\mu \mathrm{m})$ & 15 & 12 & 8 & 10 \\
* Gap-dominated area fraction, $\varphi_{g}$ & 0.40 & 0.35 & 0.45 & 0.30 \\
* Thread fiber packing density, $\alpha_{t}$ & 0.38 & 0.45 & 0.24 & 0.30 \\
* Gap fiber packing density, $\alpha_{g}$ & 0.23 & 0.32 & 0.19 & 0.24 \\
Estimated pressure drop, $\Delta P_{\text {textile }}$ & 30 & 113 & 71 & 90 \\
Estimated filtration efficiency, $E_{\text {textile }}$ & $10 \%$ & $23 \%$ & $39 \%$ & $32 \%$ \\
\hline \hline
\end{tabular}

filtration efficiencies. Therefore, they are of more use to prevent emissions of larger droplets from contagious people rather than protecting healthy individuals from submicron aerosols.

It is important to note that we only compare the mechanical filtration capabilities of mask materials in this study. For naturally charged aerosols, the image force between fibers and aerosols is not always negligible. Consequently, materials with higher dielectric constants may be more effective due to stronger electrostatic interactions with aerosols [57]. This suggests that polypropylene and polyester could be advantageous over cotton for charged aerosols. Additionally, note that the material considerations in this analysis represent only one piece of the puzzle in determining the efficacy of masks. In particular, a well-fitted mask is critical to a mask's value; masks that are well-shaped to provide an effective seal on the wearer's face and are sufficiently comfortable that the user keeps them on are likely to be worth most tradeoffs in decreased filtration efficiency [58]. Given that caveat, here we provide convenient, practical guidance for estimating the performance of cloth masks through an enhanced understanding of the permeability and aerosol filtration aspects of heterogeneous random fiber networks.

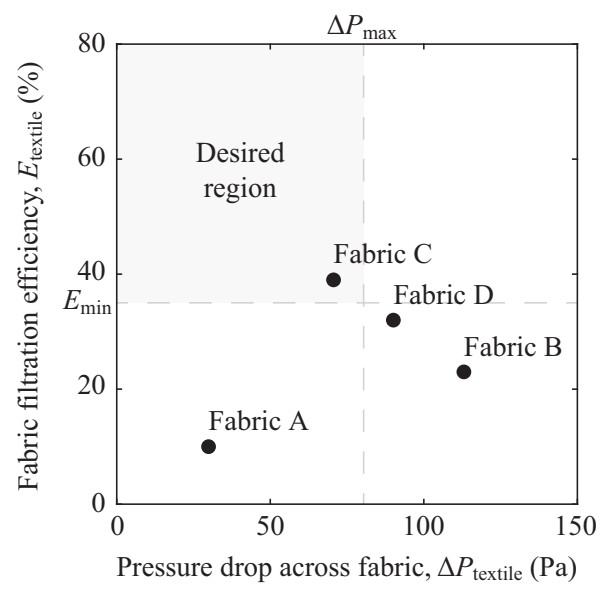

FIG. 9. Selection of mask material based on the tradeoff between filtration efficiency $E_{\text {textile }}$ and pressure drop $\Delta P_{\text {textile. }}$. 


\section{ACKNOWLEDGMENTS}

We thank Irmgard Bischofberger for helping revise the manuscript and providing valuable suggestions. We acknowledge the anonymous referees for their insightful comments, which motivated the double-porosity approach. X.M. has received support from CIMIT through the POCTRN program. CIMIT and POCTRN are supported by the RADx Tech program, and they have been funded in part with federal funds from the National Institute of Biomedical Imaging and Bioengineering, National Institutes of Health, Department of Health and Human Services, under Grant No. U54EB015408.

\section{APPENDIX A: $f(\alpha)$ AND $C_{\text {slip }}$ IN THE PRESSURE DROP MODEL}

Equations (2) and (3) represent two functional forms of $f(\alpha)$ for the calculation of permeability $\kappa$ [Eq. (1)], which are compared in Fig. 10. Additionally, we plot the pressure correction factor $C_{\text {slip }}$ as a function of fiber diameter $d_{f}$ for varying $\alpha$ values in Fig. 11 [Eq. (4)].

\section{APPENDIX B: ESTIMATING MASK PARAMETERS $\varphi_{g}, \alpha_{t}$, AND $\alpha_{g}$}

In this Appendix, we provide a strategy to estimate the gap-dominated area fraction $\left(\varphi_{g}\right)$, the thread fiber packing density $\left(\alpha_{t}\right)$, and the gap fiber packing density $\left(\alpha_{g}\right)$ from textile images. To obtain $\varphi_{g}$, we first analyze the distribution of grayscale $Y$ of a transmitted light image of the textile (Fig. 12). The initial gap-dominated region is extracted with a threshold grayscale $Y_{\text {th }}$. Because raised fuzzy fibers may not be completely counted toward the gap-dominated region, we employ an algorithm to correct the initial extraction: for a specific pixel in the textile image, if over half of its neighbors belong to gap-pixels, then this pixel is also marked as a gap-pixel; and vice versa. After repeating this procedure for many iterations, the raised fuzzy fibers will be included in the gap-dominated region, and fake gap-pixels will also be eliminated (Fig. 12).

After obtaining $\varphi_{g}$, we estimate $\alpha_{t}$ and $\alpha_{g}$ by assuming that the inverted grayscale, $Y^{*}=1-Y$, is linearly related to the local fiber packing density $\alpha$. Although a nonlinear function may describe the $\alpha-Y^{*}$ relationship more accurately, we find that the linear approximation suffices for our purposes.

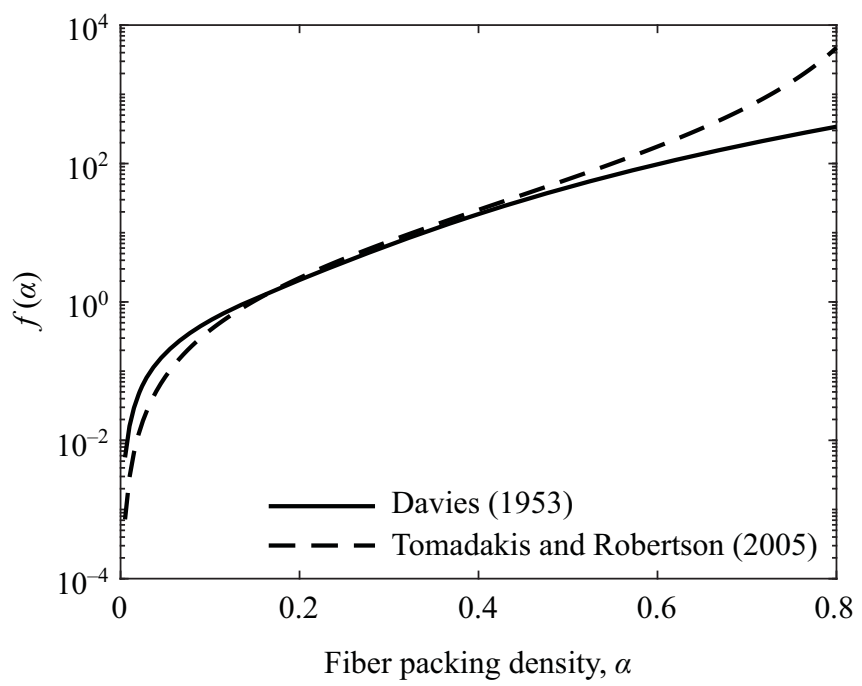

FIG. 10. Comparison of $f(\alpha)$ curves by Davies [24] and Tomadakis and Robertson [22]. 


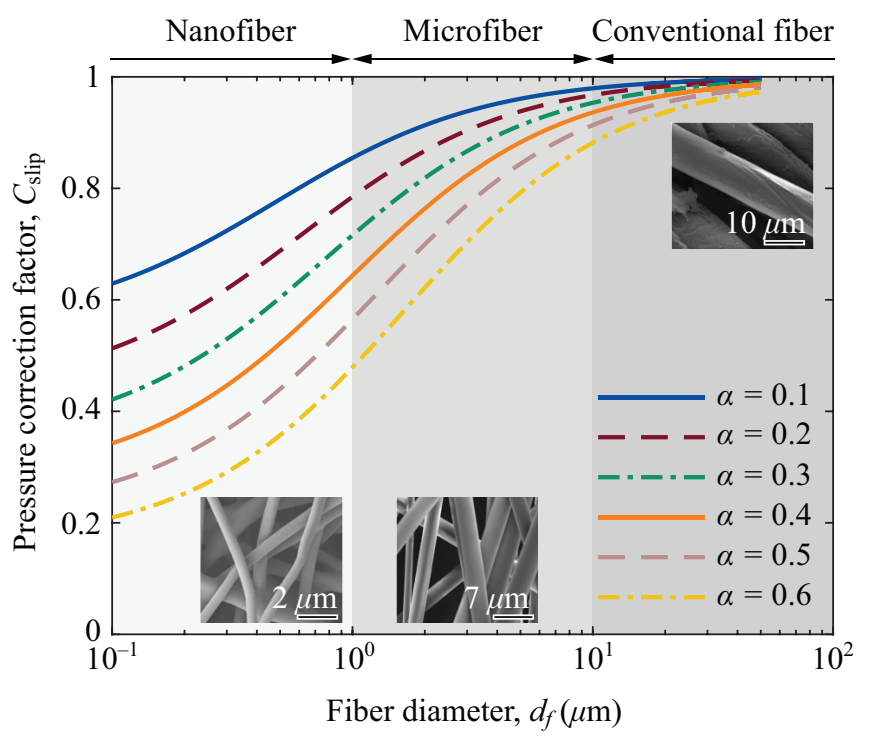

FIG. 11. Pressure correction factor $C_{\text {slip }}$ as a function of fiber diameter $d_{f}$ for varying $\alpha$ values. Microscopic images by Alarifi et al. [54] for nanofibers, Martín et al. [55] for microfibers, and Grgac et al. [56] for conventional fibers. Images of microfibers and conventional fibers are adapted under the terms of the CC-BY Creative Commons Attribution 4.0 International license.

Under our linear assumption, $\alpha_{t}$ and $\alpha_{g}$ can be calculated as

$$
\alpha_{g}=\left[\frac{\bar{Y}_{g}^{*}}{\bar{Y}_{g}^{*} \varphi_{g}+\bar{Y}_{t}^{*}\left(1-\varphi_{g}\right)}\right] \alpha \quad \text { and } \quad \alpha_{t}=\left[\frac{\bar{Y}_{t}^{*}}{\bar{Y}_{g}^{*} \varphi_{g}+\bar{Y}_{t}^{*}\left(1-\varphi_{g}\right)}\right] \alpha,
$$

where $\bar{Y}_{g}^{*}$ and $\bar{Y}_{t}^{*}$ denote the average inverted grayscale in the gap- and thread-dominated regions, respectively.

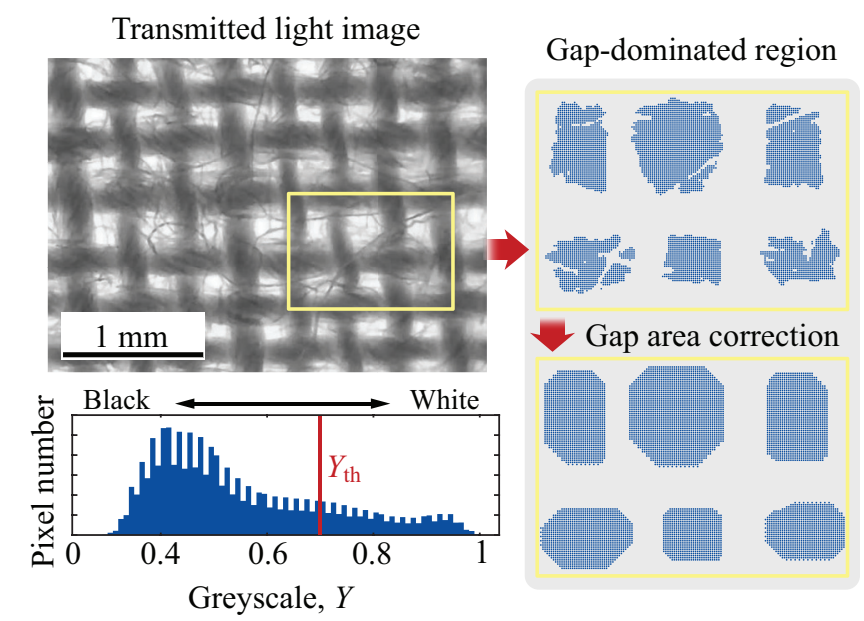

FIG. 12. Method for the estimation of gap-dominated area fraction $\varphi_{g}$. The gap-dominated region is first extracted with a threshold grayscale $Y_{\text {th }}$ from the transmitted light image of the textile. $\varphi_{g}$ is finally determined after the correction of the gap area. The textile image is adapted from Zangmeister et al. [9]. 
(a)

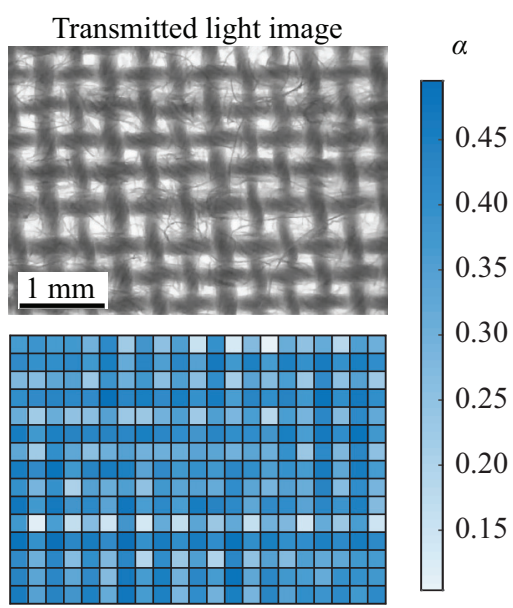

Mapped fiber packing density, $\alpha$ (b)

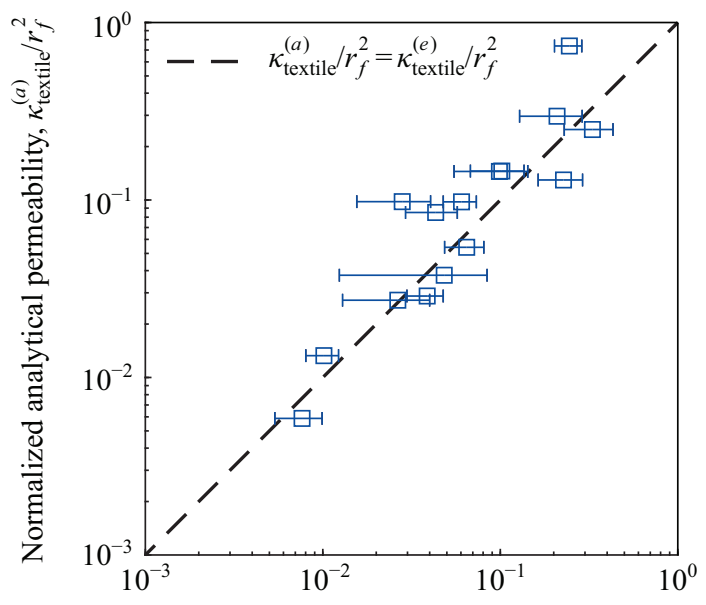

Normalized experimental permeability, $\kappa_{\text {textile }}^{(e)} / r_{f}^{2}$

FIG. 13. Permeability-map method for the estimation of $\Delta P_{\text {textile }}$. (a) Distribution of fiber packing density for a woven textile mapped on a uniform grid and (b) comparison between $\kappa_{\text {textile }}^{(e)} / r_{f}^{2}$ and $\kappa_{\text {textile }}^{(a)} / r_{f}^{2}$ for the same masks shown in Fig. 4. The textile image is adapted from Zangmeister et al. [9].

Finally, the estimated values of $\varphi_{g}, \alpha_{t}$, and $\alpha_{g}$ are influenced by the specific threshold $Y_{\mathrm{th}}$. In this study, we choose $Y_{\text {th }}$ to be the 70-percentile of the grayscale distribution. Note that other rules for the definition of $Y_{\text {th }}$ can yield different $\varphi_{g}$ values, since the gap area is ambiguous in nature for many weave styles. For a feasible rule, the estimated $\Delta P_{\text {textile }}$ should be robust against moderate variations in $Y_{\text {th }}$. Correspondingly, the textile is located at the intermediate plateau region or the homogeneous limit in Fig. 3.

\section{APPENDIX C: PERMEABILITY-MAP METHOD FOR ESTIMATING $\Delta P_{\text {textile }}$}

The transmitted light images can also be used in an alternative approach to the "double-porosity" method to estimate $\Delta P_{\text {textile }}$, termed the permeability-map method for short. In this method, we divide the textile into a uniform grid of porous media and calculate the overall permeability $\kappa_{\text {textile }}$ with the permeabilities of all elements $\kappa_{i}$, namely

$$
\kappa_{\text {textile }}=\frac{\mu U Z}{\Delta P_{\text {textile }}}=\frac{1}{N} \sum_{i=1}^{N} \kappa_{i}, \quad i=1, \ldots, N,
$$

which is a generalized version of Eq. (9). To implement Eq. (C1), we first map the transmitted light image of the textile onto a uniform grid and approximate each element to be homogeneous [Fig. 13(a)]. The grid size is chosen to be on the order of the thread pitch of the textile, such that Darcy's law is applicable to each element, and the textile is not overly homogenized. Based on the linear assumption for the $\alpha-Y^{*}$ relationship, we extract the average fiber packing density $\alpha_{i}$ for each element in a similar manner as in Appendix B. The permeability $\kappa_{i}$ is then calculated to be $\kappa_{i}=d_{f}^{2} /\left[4 f\left(\alpha_{i}\right)\right]$. After obtaining $\kappa_{i}$, we can further estimate $\kappa_{\text {textile }}$ and $\Delta P_{\text {textile }}$ with Eq. $(\mathrm{C} 1)$.

Based on the permeability-map method, we present in Fig. 13(b) the predicted normalized permeabilities $\kappa_{\text {textile }}^{(a)} / r_{f}^{2}$ for the same group of masks shown in Fig. 4. Similar to the "double-porosity" results, our predictions generally agree with experimental results $\kappa_{\text {textile }}^{(e)} / r_{f}^{2}$. 
[1] K. A. Prather, C. C. Wang, and R. T. Schooley, Reducing transmission of SARS-CoV-2, Science 368, $1422(2020)$.

[2] N. H. L. Leung, D. K. W. Chu, E. Y. C. Shiu, K.-H. Chan, J. J. McDevitt, B. J. P. Hau, H.-L. Yen, Y. Li, D. K. M. Ip, J. S. M. Peiris, W.-H. Seto, G. M. Leung, D. K. Milton, and B. J. Cowling, Respiratory virus shedding in exhaled breath and efficacy of face masks, Nat. Med. 26, 676 (2020).

[3] CDC, Use of masks to help slow the spread of COVID-19, https://www.cdc.gov/coronavirus/2019-ncov/ prevent-getting-sick/diy-cloth-face-coverings.html (accessed Sep 02, 2020).

[4] R. Thakur, D. Das, and A. Das, Electret air filters, Sep. Purif. Rev. 42, 87 (2013).

[5] WHO, Advice on the use of masks in the context of COVID-19, https://www.who.int/publications-detailredirect/advice-on-the-use- of-masks-in-the-community-during-home-care-and-in-healthcare-settingsin-the-context-of-the-novel-coronavirus-(2019-ncov)-outbreak (accessed Sep 02, 2020).

[6] A. Davies, K.-A. Thompson, K. Giri, G. Kafatos, J. Walker, and A. Bennett, Testing the efficacy of homemade masks: Would they protect in an influenza pandemic? Disaster Med. Public Health Preparedness 7, 413 (2013).

[7] S. Rengasamy, B. Eimer, and R. E. Shaffer, Simple respiratory protection/evaluation of the filtration performance of cloth masks and common fabric materials against 20-1000 nm size particles, Ann. Occup. Hyg. 54, 789 (2010).

[8] M. Zhao, L. Liao, W. Xiao, X. Yu, H. Wang, Q. Wang, Y. L. Lin, F. S. Kilinc-Balci, A. Price, L. Chu, M. C. Chu, S. Chu, and Y. Cui, Household materials selection for homemade cloth face coverings and their filtration efficiency enhancement with triboelectric charging, Nano Lett. 20, 5544 (2020).

[9] C. D. Zangmeister, J. G. Radney, E. P. Vicenzi, and J. L. Weaver, Filtration efficiencies of nanoscale aerosol by cloth mask materials used to slow the spread of SARS-CoV-2, ACS Nano 14, 9188 (2020); see the experimental data in the supporting information at https://pubs.acs.org/doi/suppl/10.1021/acsnano. 0c05025/suppl_file/nn0c05025_si_002.pdf. Further permissions related to the material excerpted should be directed to the ACS Publications.

[10] S. R. Lustig, J. J. H. Biswakarma, D. Rana, S. H. Tilford, W. Hu, M. Su, and M. S. Rosenblatt, Effectiveness of common fabrics to block aqueous aerosols of virus-like nanoparticles, ACS Nano 14, 7651 (2020).

[11] A. Konda, A. Prakash, G. A. Moss, M. Schmoldt, G. D. Grant, and S. Guha, Aerosol filtration efficiency of common fabrics used in respiratory cloth masks, ACS Nano 14, 6339 (2020).

[12] H.-w. Cho, C.-S. Yoon, J.-H. Lee, S.-j. Lee, A. Viner, and E. W. Johnson, Comparison of pressure drop and filtration efficiency of particulate respirators using welding fumes and sodium chloride, Ann. Occup. Hyg. 55, 666 (2011).

[13] K. M. Shakya, A. Noyes, R. Kallin, and R. E. Peltier, Evaluating the efficacy of cloth face- masks in reducing particulate matter exposure, J. Exposure Sci. Environ. Epidemiol. 27, 352 (2017).

[14] W. Mueller, C. J. Horwell, A. Apsley, S. Steinle, S. McPherson, J. W. Cherrie, and K. S. Galea, The effectiveness of respiratory protection worn by communities to protect from volcanic ash inhalation. Part I: Filtration efficiency tests, Int. J. Hyg. Environ. Health 221, 967 (2018).

[15] J. W. Cherrie, A. Apsley, H. Cowie, S. Steinle, W. Mueller, C. Lin, C. J. Horwell, A. Sleeuwenhoek, and M. Loh, Effectiveness of face masks used to protect Beijing residents against particulate air pollution, Occup. Environ. Med. 75, 446 (2018).

[16] B. B. Neupane, S. Mainali, A. Sharma, and B. Giri, Optical microscopic study of surface morphology and filtering efficiency of face masks, PeerJ 7, e7142 (2019).

[17] O. Aydin, B. Emon, S. Cheng, L. Hong, L. P. Chamorro, and M. T. A. Saif, Performance of fabrics for home-made masks against the spread of COVID-19 through droplets: A quantitative mechanistic study, Extreme Mech. Lett. 40, 100924 (2020).

[18] E. P. Fischer, M. C. Fischer, D. Grass, I. Henrion, W. S. Warren, and E. Westman, Low- cost measurement of face mask efficacy for filtering expelled droplets during speech, Sci. Adv. 6, eabd3083 (2020).

[19] B. Maxted, Dust masks for indian quarry workers: A comparative analysis of the filtering efficiency of fabrics, J. Humanitarian Eng. 1, 15 (2011).

[20] A. S. Sangani and C. Yao, Transport processes in random arrays of cylinders. II. Viscous flow, Phys. Fluids 31, 2435 (1988). 
[21] A. Tamayol and M. Bahrami, Transverse permeability of fibrous porous media, Phys. Rev. E 83, 046314 (2011).

[22] M. M. Tomadakis and T. J. Robertson, Viscous permeability of random fiber structures: Comparison of electrical and diffusional estimates with experimental and analytical results, J. Compos. Mater. 39, 163 (2005).

[23] L. Spielman and S. L. Goren, Model for predicting pressure drop and filtration efficiency in fibrous media, Environ. Sci. Technol. 2, 279 (1968).

[24] C. Davies, The separation of airborne dust and particles, Proc. Inst. Mech. Eng. 167, 185 (1953).

[25] M. Tahir and H. Vahedi Tafreshi, Influence of fiber orientation on the transverse permeability of fibrous media, Phys. Fluids 21, 083604 (2009).

[26] O. Rahli, L. Tadrist, M. Miscevic, and R. Santini, Fluid flow through randomly packed monodisperse fibers: the Kozeny-Carman parameter analysis, J. Fluids Eng. 119, 188 (1997).

[27] G. W. Jackson and D. F. James, The permeability of fibrous porous media, Can. J. Chem. Eng. 64, 364 (1986).

[28] A. Goodings, Air flow through textile fabrics, Text. Res. J. 34, 713 (1964).

[29] A. Kulichenko and L. V. Langenhove, The resistance to flow transmission of porous materials, J. Text. Inst. 83, 127 (1992).

[30] C. Brasquet and P. Le Cloirec, Pressure drop through textile fabrics: experimental data modelling using classical models and neural networks, Chem. Eng. Sci. 55, 2767 (2000).

[31] Q. Wang, B. Maze, H. V. Tafreshi, and B. Pourdeyhimi, On the pressure drop modeling of monofilamentwoven fabrics, Chem. Eng. Sci. 62, 4817 (2007).

[32] C. N. Davies, Air Filtration (Academic, London, 1973).

[33] W. C. Hinds, Aerosol Technology: Properties, Behavior, and Measurement of Airborne Particles (Wiley, New York, 1999).

[34] Y. Liu, Z. Ning, Y. Chen, M. Guo, Y. Liu, N. K. Gali, L. Sun, Y. Duan, J. Cai, D. Westerdahl et al., Aerodynamic analysis of SARS-CoV-2 in two wuhan hospitals, Nature (London) 582, 557 (2020).

[35] H. P. G. Darcy, Les Fontaines Publiques de la Ville de Dijon. Exposition et Application des Principes Suivre et des Formules Employer dans les Questions de Distribution d'eau, etc (Dalamont, Paris, 1856).

[36] D. Thomas, A. Charvet, N. Bardin-Monnier, and J.-C. Appert-Collin, Aerosol Filtration (Elsevier, Amsterdam, 2016).

[37] A. Kirsch, I. Stechkina, and N. Fuchs, Effect of gas slip on the pressure drop in fibrous filters, J. Aerosol Sci. 4, 287 (1973).

[38] J. Pich, Pressure drop of fibrous filters at small Knudsen numbers, Ann. Occup. Hyg. 9, 23 (1966).

[39] J. Pich, Pressure characteristics of fibrous aerosol filters, J. Colloid Interface Sci. 37, 912 (1971).

[40] R. C. Brown, Air Filtration: An Integrated Approach to the Theory and Applications of Fibrous Filters (Pergamon, Oxford, 1993).

[41] A. Podgorski, A. Bałazy, and L. Gradon, Application of nanofibers to improve the filtration efficiency of the most penetrating aerosol particles in fibrous filters, Chem. Eng. Sci. 61, 6804 (2006).

[42] J. Wang, S. C. Kim, and D. Y. Pui, Investigation of the figure of merit for filters with a single nanofiber layer on a substrate, J. Aerosol Sci. 39, 323 (2008).

[43] S. Hosseini and H. V. Tafreshi, Modeling permeability of 3-D nanofiber media in slip flow regime, Chem. Eng. Sci. 65, 2249 (2010).

[44] Y.-Y. Kuo, F. C. Bruno, and J. Wang, Filtration performance against nanoparticles by electrospun nylon-6 media containing ultrathin nanofibers, Aerosol Sci. Technol. 48, 1332 (2014).

[45] S. Chattopadhyay, T. A. Hatton, and G. C. Rutledge, Aerosol filtration using electrospun cellulose acetate fibers, J. Mater. Sci. 51, 204 (2016).

[46] K. Lee and B. Liu, Theoretical study of aerosol filtration by fibrous filters, Aerosol Sci. Technol. 1, 147 (1982).

[47] A. A. Kirsch and N. A. Fuchs, Studies on fibrous aerosol filters. III. Diffusional deposition of aerosols in fibrous filters, Ann. Occup. Hyg. 11, 299 (1968). 
[48] B. Y. Liu and K. L. Rubow, Efficiency, pressure drop and figure of merit of high efficiency fibrous and membrane filter media, in Proceedings of the Fifth World Filtration Congress (Scientific Research Publisher, Nice, France, 1990), Vol. 3, pp. 112-119.

[49] A. Jackiewicz and L. Werner, Separation of nanoparticles from air using melt-blown filtering media, Aerosol Air Qual. Res. 15, 2422 (2015).

[50] K. Lee and B. Liu, On the minimum efficiency and the most penetrating particle size for fibrous filters, J. Air Pollut. Control Assoc. 30, 377 (1980).

[51] G. Karniadakis, A. Beskok, and N. Aluru, Microflows and Nanoflows: Fundamentals and Simulation (Springer Science \& Business Media, New York, 2006).

[52] P. Li, C. Wang, Y. Zhang, and F. Wei, Air filtration in the free molecular flow regime: a review of highefficiency particulate air filters based on carbon nanotubes, Small 10, 4543 (2014).

[53] See ASTM F2100-19: "Standard specification for performance of materials used in medical face masks" at https://compass.astm.org/Standards/HISTORICAL/F2100-19.htm for testing standards. $\Delta P_{\max }=50 \mathrm{~Pa}$ is calculated at the reduced velocity $U=0.053 \mathrm{~m} / \mathrm{s}$.

[54] I. M. Alarifi, W. S. Khan, and R. Asmatulu, Synthesis of electrospun polyacrylonitrile-derived carbon fibers and comparison of properties with bulk form, PLoS One 13, e0201345 (2018).

[55] D. Morillo Martín, M. Magdi Ahmed, M. Rodríguez, M. A. García, and M. Faccini, Aminated polyethylene terephthalate (PET) nanofibers for the selective removal of $\mathrm{Pb}$ (II) from polluted water, Materials $\mathbf{1 0}$, 1352 (2017).

[56] S. Flinčec Grgac, A. Tarbuk, T. Dekanić, W. Sujka, and Z. Draczyński, The chitosan implementation into cotton and polyester/cotton blend fabrics, Materials 13, 1616 (2020).

[57] M. Alonso, F. Alguacil, J. Santos, N. Jidenko, and J. Borra, Deposition of ultrafine aerosol particles on wire screens by simultaneous diffusion and image force, J. Aerosol Sci. 38, 1230 (2007).

[58] P. W. Clapp, E. E. Sickbert-Bennett, J. M. Samet, J. Berntsen, K. L. Zeman, D. J. Anderson, D. J. Weber, and W. D. Bennett, Evaluation of cloth masks and modified procedure masks as personal protective equipment for the public during the COVID-19 pandemic, JAMA Internal Medicine 181, 463 (2021). 